\title{
VARIACIÓN DIURNA DEL CORTISOL Y SU RELACIÓN CON ESTRÉS, OPTIMISMO Y ESTRATEGIAS DE AFRONTAMIENTO EN MUJERES CON CÁNCER DE MAMA
}

\author{
Dehisy Marisol Juárez García*1,2 , René Landero Hernández ${ }^{2}$, Mónica Teresa González Ramírez ${ }^{2}$, Leticia Jaime Bernal ${ }^{1}$ \\ Universidad Autónoma de Nuevo León, Centro de Investigación y Desarrollo en Ciencias de la Salud ${ }^{1}$, Facultad de Psicología ${ }^{2}$ \\ Recibido, febrero $12 / 2015$ \\ Concepto evaluación, octubre 19/2015 \\ Aceptado, noviembre 6/2015 \\ Referencia: Juárez García, D.M., Landero Hernández, R., Gon-
zález Ramírez, M.T. \& Jaime Bernal, L. (2016). Variación diurna
del cortisol y su relación con estrés, optimismo y estrategias de
afrontamiento en mujeres con cáncer de mama. Acta Colombiana
de Psicología, 19(1), 103-112. DOI: 10.14718/ACP.2016.19.1.6
}

Resumen

\begin{abstract}
El ritmo diurno del cortisol se ha visto alterado en los pacientes con cáncer. Factores como el avance de la enfermedad y los niveles de estrés se han considerado para explicar esta condición; sin embargo, los resultados no son claros. El objetivo de este estudio fue evaluar y determinar si existen diferencias en los niveles de cortisol en mujeres con cáncer de mama en diferentes estadios, y analizar la relación entre los niveles de cortisol y el estrés, el optimismo y las estrategias de afrontamiento. Se recolectaron muestras de cortisol salival durante dos días, y se aplicaron cuestionarios psicológicos de estrés percibido (PSS), optimismo disposicional (LOT-R) y estrategias de afrontamiento (MAC) a 17 mujeres con cáncer de mama en estadio I, II y III. Los resultados muestran que las pacientes con cáncer de mama en los tres estadios presentan un ritmo diurno de cortisol normal y no difieren significativamente en los niveles de cortisol. Respecto a las variables psicológicas, solo difieren en la variable de optimismo. Las estrategias de afrontamiento - espíritu de lucha y evitación cognitiva - son las variables que tienen más influencia en los niveles de cortisol, y explican un 55\% de la varianza. Se discuten las implicaciones de estos resultados. Palabras Clave: cortisol, estrés, ritmo diurno del cortisol, estrategias de afrontamiento.
\end{abstract}

\section{DIURNAL CORTISOL VARIATION AND ITS RELATIONSHIP WITH STRESS AND COPING STRATEGIES IN WOMEN WITH BREAST CANCER}

\begin{abstract}
Diurnal cortisol rhythm is affected in patients with cancer. Factors such as disease progression and stress levels are regarded as possible causes of this condition, however results are not clear. The aim of this study was to assess and determine whether there are differences in cortisol levels in women with breast cancer in different stages and analyze the relationship between cortisol levels and stress, optimism and coping strategies. Salivary cortisol samples were collected for two days and psychological questionnaires of perceived stress (PSS), dispositional optimism (LOT-R) and coping strategies (MAC) were administered to 17 breast cancer women in stage I, II and III of the disease. The results show that patients with breast cancer in the three stages have a normal diurnal cortisol rhythm and do not differ significantly in cortisol levels. Regarding the psychological variables, they differ only in the optimism. Coping strategies such as fighting spirit and cognitive avoidance are the variables that have more influence on the cortisol levels, explaining $55 \%$ of variance. The implications of these results are discussed.

Key Words: cortisol, stress, diurnal cortisol rhythm, coping strategies.
\end{abstract}

* Universidad Autónoma de Nuevo León. Centro de Investigación y Desarrollo en Ciencias de la Salud. Unidad de Psicología de la Salud. Av. Carlos Canseco s/n con Av. Gonzalitos, Mitras Centro Monterrey, Nuevo León, México, C.P. 64460. Teléfono (52 81 ) 13404370 Ext. 1768. dehisy_jg@yahoo.com.mx, dehisy.juarezg@uanl.mx

Este artículo se deriva de la Tesis Doctoral: "Ritmo diurno del cortisol y su relación con variables psicológicas en mujeres con cáncer de mama". Agradecemos a los doctores Manuel García Solís y Jorge Martínez Rodríguez por el apoyo brindado en la consecución de los pacientes. Igualmente, al Maestro Arnoldo Téllez López y al doctor Carlos Medina de la Garza por el respaldo administrativo para este proyecto. 


\title{
VARIAÇÃO DIURNA DO CORTISOL E SUA RELAÇÃO COM O ESTRESSE: OTIMISMO E ESTRATÉGIAS DE ENFRENTAMENTO EM MULHERES COM CÂNCER DE MAMA
}

\begin{abstract}
Resumo
O ritmo diurno do cortisol vem sendo visto alterado nos pacientes com câncer. Fatores como o avanço da doença e os níveis de estresse estão sendo considerados para explicar essa condição; contudo, os resultados não são claros. O objetivo deste estudo foi avaliar e determinar se existem diferenças nos níveis de cortisol em mulheres com câncer de mama de diferentes estágios, bem como analisar estratégias de enfrentamento. Foram coletadas amostras de cortisol salival durante dois dias e aplicados questionários psicológicos de estresse percebido, otimismo disposicional e estratégias de enfrentamento a 17 mulheres com câncer de mama em estágio I, II e III. Os resultados mostram que as pacientes com esse câncer nos três estágios apresentam um ritmo diurno de cortisol normal e não se diferenciam significativamente na variável de otimismo. A respeito das variáveis psicológicas, só diferem na variável de otimismo. As estratégias de enfrentamento — espírito de luta e evitação cognitiva— são as variáveis que têm mais influência nos níveis de cortisol e explicam $55 \%$ da variação. Discutem-se as implicações desses resultados.

Palavras-chave: cortisol, estresse, ritmo diurno do cortisol, estratégias de enfrentamento.
\end{abstract}

\section{INTRODUCCIÓN}

El cortisol es un glucocorticoide de la corteza adrenal con efectos en el metabolismo de proteínas, carbohidratos y lípidos. Los niveles de concentración del cortisol son altos en horas muy tempranas de la mañana (6am-8am), y presentan una actividad mínima en la tarde (4pm-6pm). Considerado un ritmo circadiano (Fischbach y Dunning, 2009; Kronfol, Nair, Zhang, Hill y Brown, 1997), este patrón se presenta en personas sanas. Los cambios en el cortisol también dependen de la activación del eje Hipotalámico Pituitario Adrenal (HPA), que responde de manera homeostática ante diferentes desafíos estresantes. La activación constante debida a un estrés crónico puede generar un desbalance bioquímico y deterioro en diferentes sistemas del cuerpo, como el inmunológico, incrementando la vulnerabilidad a enfermedades, y el sistema neuroendocrino que altera los ritmos circadianos (Moscoso, 2009; Sephton y Spiegel, 2003; Young y Welsh, 2005).

El estrés y el cáncer se relacionan desde el momento en que una persona se siente vulnerable a padecer dicha enfermedad; los efectos de esta percepción de riesgo y la incertidumbre que conlleva el diagnóstico se observan en la respuesta biológica y psicológica del estrés (Barroilhet, Forjazy Garrido, 2005; Dettenborn, James, Valdimarsdottir, Montgomery y Bovbjerg, 2006; Lang, Berbaum y Lutgendorf, 2009). La respuesta del estrés también depende de variables psicológicas llamadas estrategias de afrontamiento, y sus efectos se observan en la modulación de la respuesta al estrés a través de mecanismos que la persona utiliza para hacerle frente a las situaciones que evalúa como estresantes, disminuyendo la repuesta biológica del organismo a través de: 1) menor percepción o menor sentimiento de vulnerabilidad de la amenaza y 2) un manejo más efectivo cuando la amenaza es detectada (Taylor et al., 2008).

La interacción de estas variables con el cortisol es importante debido a que pacientes con diferentes tipos de cáncer presentan una alteración de la función circadiana endocrina, la cual se observa principalmente en valores bajos y menor variabilidad de los niveles del cortisol (Touitou, Bogdan, Lévi, Benavides y Auzéby, 1996; Abercrombie et al., 2006; Mazzoccoli, Vendemiale, De Cata, Carughi y Tarquini, 2010, Mazzoccoli, Tarquini, Durfot y Francoise, 2011; Weinrib et al., 2010). Estas alteraciones se han relacionado con periodos cortos de sobrevivencia (Septhon, Sapolsky, Kraemer y Spiegel 2000; Septhon et al., 2012).

Sin embargo, otros estudios muestran que los pacientes con cáncer presentan un ritmo diurno del cortisol normal comparable al de personas sanas (Haus et al., 2001; Garland et al. 2004; Vedhara, Tuin, Miles, Sanderman y Ranchor, 2006; Carlson et al., 2007; Mazzoccoli, Giuliani y Sothern, 2012). Asimismo, las variables que han sido consideradas para explicar la alteración del ritmo diurno, como el avance de la enfermedad, son contradictorios o en el caso del estrés, los resultados son escasos o nulos (Touitou et al., 1995; Porter et al., 2003; Nakaya et al., 2005; Garland et al., 2004; Carlson et al., 2007; Vedhara et al., 2003, 2006). Por esta razón, el objetivo de este estudio fue comparar los niveles de cortisol en mujeres con cáncer de mama de diferentes estadios de la enfermedad, y examinar la relación entre los niveles de cortisol y el estrés, el optimismo y las estrategias de afrontamiento. 


\section{MÉTODO}

\section{Tipo de estudio}

Se utilizó un diseño no experimental con una muestra no probabilística (por conveniencia).

\section{Participantes}

Las pacientes con cáncer de mama fueron referidas por un oncólogo y contactadas en la Clínica de mama del Hospital Bernardo Sepúlveda. Se incluyeron las mujeres diagnosticadas con cáncer de mama programadas a quimioterapia. Se excluyeron las pacientes con cáncer previo o cáncer bilateral. Se eliminaron los datos con muestras incompletas o contaminadas. De 25 pacientes que aceptaron participar, dos no asistieron y tres fueron excluidas. De 20 pacientes evaluadas, tres fueron eliminadas.

Se analizaron los datos de 17 mujeres con cáncer de mama con edad promedio de 50.65 años $(\mathrm{DE}=8.3)$. Once de ellas eran casadas; tres, solteras; dos vivían en unión libre, y una era viuda. Solo cuatro de ellas trabajaban en el momento del estudio. 14 habían tenido cirugía, y tres no. Referente al estadio de la enfermedad, seis de ellas se encontraban en el estadio I; seis, en el estadio II; cuatro, en estadio III, y un dato no estaba disponible.

Instrumentos

Método Oral Swab (Salimetrics $\left.{ }^{\circledR}\right)$ : Fue utilizado para recolectar las muestras del cortisol salival. Consiste en colocar y mantener por dos minutos un copo de algodón bajo la lengua, y posteriormente depositarlo en un tubo de almacenamiento. Este método es adecuado y fácil de usar para la recolección de cortisol (Salimetrics, 2012).

Escala de Estrés Percibido: Elaborada por Cohen, Kamarak y Mermelstein (1983) para evaluar el grado en que las situaciones de la vida son valoradas como estresantes. En este estudio se utilizó la adaptación cultural realizada en México por González y Landero (2007), la cual cuenta con 14 ítems y un coeficiente de alfa de Cronbach de 0.83 . El alfa de Cronbach obtenido en esta muestra fue de 0.86 . La puntuación va de nunca -0- a muy a menudo -4- con una puntación total en un rango de 0 a 56. La mayor puntuación corresponde a mayor estrés percibido.

Life Orientation Test (Scheier, Carver y Bridges, 1994): Evalúa las diferencias individuales generalizadas de optimismo contra pesimismo. Se utilizó la versión al español de Otero, Luengo, Romero, Gómez y Castro (1998), la cual cuenta con diez ítems y un alfa de Cronbach de.79. En esta muestra el alfa de Cronbach fue de 0.64. Las puntuaciones de los ítems redactados en sentido negativo se invierten y se obtiene una puntuación total orientada hacia el polo de optimismo, cuyo recorrido es de 6 a 30. Una puntuación alta indica mayor optimismo.
La Escala de Ajuste Mental al Cáncer. Fue desarrollada para evaluar el grado en que los pacientes adoptan respuestas para adaptarse al diagnóstico de cáncer y su tratamiento. Se utilizó la traducción al español de Caro e Ibáñez (Watson et al., 1988) que cuenta con 40 ítems divididos en cinco subescalas: esperanza-desesperanza, ansiedad-preocupación, espíritu de lucha, evitación cognitiva y fatalismo, las cuales tienen un rango de puntaje de 6 a 24, 9 a 36,16 a 64,1 a 4 y 8 a 32, respectivamente. Ha obtenido un alfa de Cronbach de 0.84; en esta muestra el alfa fue de 0.64 .

\section{Procedimiento}

Este estudio se realizó en tres etapas: Etapa 1: Luego de la autorización de los comités de bioética de las instituciones: Hospital Bernardo Sepúlveda de la Secretaría de Salud y el Centro de Investigación de Desarrollo en Ciencias de la Salud, (CIDICS) UANL, se asistió a la Clínica de Mama del Hospital para contactar a las pacientes; a quienes cumplían con los criterios, se les realizaba una llamada para invitarlas y explicarles el estudio; a las pacientes que aceptaban, se les agendaba una cita.

Etapa 2: Las pacientes que aceptaban participar firmaban el consentimiento informado y pasaban dos días consecutivos en la Unidad de Psicología del CIDICS, durante los cuales se recolectaron seis muestras de saliva en los siguientes horarios: antes de dormir, al despertar, 30 y 60 minutos después de despertar, a las 12 y 16 horas. Se respetaba el horario regular de dormir y despertar de las pacientes para detectar el ritmo diurno de manera natural. Durante la recolección de datos se indicó y se supervisó que evitaran lavarse los dientes, consumir chicles, alimentos o bebidas altos en azúcar o ácido, y consumir alcohol doce horas antes de la recolección (Salimetrics, 2012). Los datos psicológicos se evaluaron durante los dos días después del desayuno. Etapa 3: Las muestras de saliva fueron etiquetadas y congeladas $\left(-20^{\circ} \mathrm{C}\right)$ hasta ser enviadas al laboratorio de Salimetrics para su análisis.

\section{Análisis de datos}

Los niveles de cortisol fueron determinados por un inmunoensayo enzimático realizado por Salimetrics, Inc. La sensibilidad del ensayo está en un rango de de 0 a 0.007 $\mu \mathrm{g} / \mathrm{dL}$. Los rangos de los coeficientes de variación del intra-ensayo son de 4 a $7 \%$ y del inter-ensayo de 3 a $11 \%$ (Salimetrics, 2013).

Los datos fueron capturados y analizados con el programa SPSS. Se realizó la Prueba de Normalidad Shapiro- Wilk en la cual fueron significativas cuatro muestras de cortisol $(\mathrm{p}<.01)$ y los puntajes de las subescalas de espíritu de lucha y evitación cognitiva $(\mathrm{p}<.01)$; debido a esto se decidió utilizar pruebas no paramétricas. Para analizar el ritmo diurno del cortisol se obtuvieron las medias de cada muestra salival, 
y se realizó una gráfica con el fin de observar los cambios de secreción del cortisol. Para validar dichos cambios en el tiempo se realizó la prueba de Friedman.

Los niveles de cortisol se obtuvieron a través del indicador del Área Bajo la Curva (AUC, por su sigla en inglés) el cual se usa en endocrinología para estimar los cambios hormonales y evaluar la secreción total durante un periodo de tiempo. Su fórmula está basada en el método trapezoide que consiste en la suma de triángulos y rectángulos basados en la información obtenida de las medidas repetidas. El cálculo de la AUC permite simplificar el análisis estadístico sin sacrificar mediciones, y permite incrementar el poder de las pruebas reduciendo la necesidad de ajustar el nivel de significancia. Para este estudio se obtuvieron dos indicadores de AUC: a) el área bajo la curva con respecto a la base (AUCg) es aquella que considera todas las mediciones y representa la concentración total del cortisol y b) y el área bajo la curva con respecto al incremento (AUCi), que se calcula con referencia a la medición de línea de base, no tiene en cuenta la distancia de cero para todas las mediciones, y representa el incremento del cortisol a través el tiempo (Fekedulegn et al., 2007; Pruessner, Kirshbaum, Meinlschmid y Hellhammer, 2003). Para evaluar las diferencias por estadio de los niveles de cortisol (AUC) y las variables psicológicas, se utilizó la prueba de Kruskal-Wallis.
Para el análisis de correlación se utilizó el coeficiente de correlación de Spearman. Por último, se llevó a cabo el análisis de regresión múltiple con el método de pasos sucesivos para evaluar las variables que explican la variable dependiente (AUCg y AUCi de cortisol).

\section{RESULTADOS}

En este apartado se presentan los resultados de acuerdo con los objetivos planteados en este estudio.

Análisis del ritmo diurno del cortisol por estadio de cáncer

Se obtuvo la media de cada muestra de cortisol por día; los datos indican que los tres estadios analizados (I, II, III) muestran un ritmo diurno del cortisol normal durante los dos días (Figura 1), presentando un incremento de la muestra antes de dormir (1) a la muestra tomada al despertar (2) $\mathrm{y}$ un decremento en la secreción del cortisol a partir de la muestra tomada media hora después de despertar (3). Estas variaciones en las concentraciones de cortisol fueron significativas para los tres estadios durante el día 1: estadio I $($ Friedman $=24.4, p=.000)$, estadio II (Friedman $=23.5$, $\mathrm{p}=.000)$ y estadio III (Friedman $=18.2, \mathrm{p}=.003)$ y durante el día 2: estadio I (Friedman $=26.6, p=.000)$, estadio II (Friedman $=19.3, \mathrm{p}=.002)$ y estadio III (Friedman $=17.4$, $\mathrm{p}=.004)$. Estos datos validan la variación diurna en los tres estadios.

\section{Día 1}

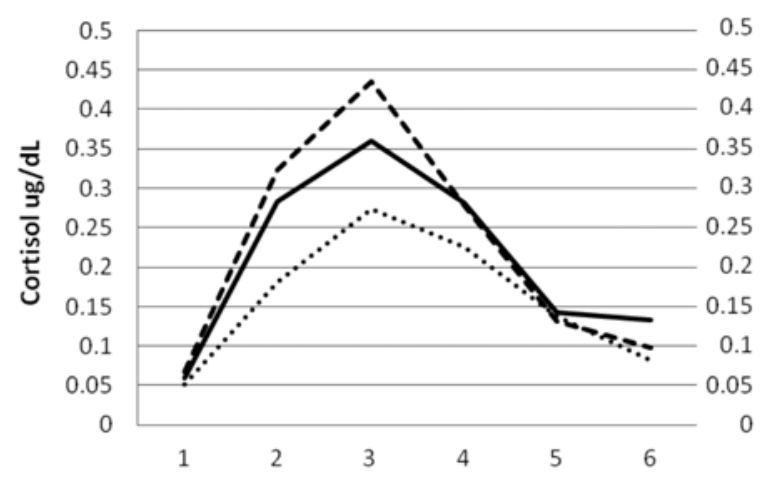

Día 2

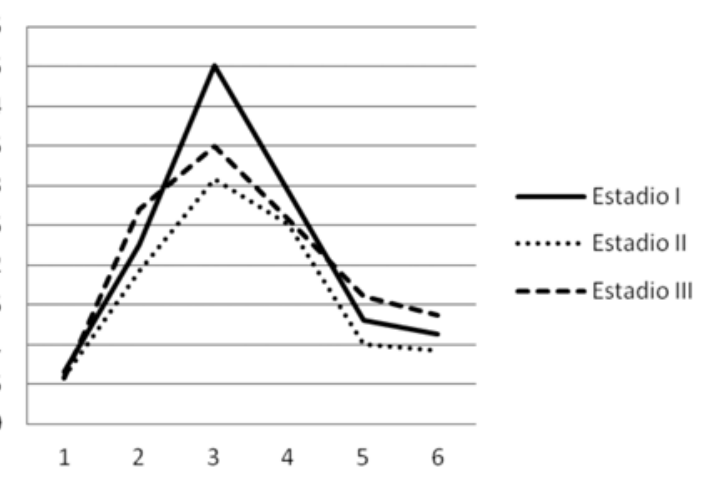

Figura 1. Ritmo diurno del cortisol salival por estadios

Nota: 1. Antes de dormir, 2. Al despertar, 3. 30 min después de despertar, 4. 60 minutos después de despertar, 5. 12h, 6 16h. Estadio I $n=6$, estadio II $n=6$ y estadio III $n=4$.

Diferencia en los niveles de cortisol y variables psicológicas según estadio del cáncer

Se analizaron estadios I, II y III comparando el AUCg y AUCi del cortisol sin obtenerse diferencias significativas.
Respecto a las variables psicológicas, solo el optimismo fue significativa (Tabla 1). 
Tabla 1.

Análisis de diferencia en los niveles de cortisol y variables psicológicas según estadio del cáncer

\begin{tabular}{lcccc}
\hline Variable & Estadio I & Estadio II & Estadio III & Kruskal-Wallis \\
Media(DE) & $n=6$ & $n=6$ & $n=4$ & Ch2 $(p)$ \\
\hline AUCg1 & $183.4(46.9)$ & $136.8(51.4)$ & $192.6(58.9)$ & $3.01(221)$ \\
AUCg2 & $167.8(38.6)$ & $139.0(57.4)$ & $180.6(66.7)$ & $1.69(429)$ \\
AUCi1 & $122.9(47.6)$ & $82.9(47.5)$ & $124.0(47.8)$ & $3.11(210)$ \\
AUCi2 & $100.5(39.9)$ & $78.6(61.3)$ & $122.2(50.7)$ & $1.83(339)$ \\
Estrés & $18(4.8)$ & $26.5(11.9)$ & $29.2(6.2)$ & $4.08(.130)$ \\
Optimismo & $43.1(4.3)$ & $38.5(6.1)$ & $35(1.8)$ & $7.40(.025)^{*}$ \\
Espíritu de & $59.0(2.4)$ & $53.6(5.2)$ & $53(5.4)$ & $3.93(.140)$ \\
lucha & $7.8(1.7)$ & $9.1(3.4)$ & $11.0(3.5)$ & $2.31(.315)$ \\
Desesperanza & $23.0(3.7)$ & $24.3(5.4)$ & $28(6.9)$ & $1.57(.456)$ \\
Ansiedad & $18.0(4.0)$ & $21.5(4.1)$ & $21.5(5.9)$ & $1.59(.451)$ \\
Fatalismo & $2.0(1.5)$ & $2.0(1.5)$ & $2.2(.95)$ & $.291(.864)$ \\
Evitación & & & &
\end{tabular}

Relación entre niveles de cortisol AUC y variables psicológicas

Para relacionar las variables psicológicas con los niveles de cortisol se obtuvo la media de la AUC respecto a la base (AUC g) y la media de la AUC respecto al incremento (AUCi). Como se observa en la Tabla 2, el AUCg del cortisol se correlacionó negativa y significativamente con estrés, es decir, a mayor estrés, menor secreción total del cortisol.
El AUCg se correlacionó positiva y significativamente con la estrategia de afrontamiento de espíritu de lucha y evitación, lo que indica que a mayor uso de las estrategias de espíritu de lucha y evitación cognitiva, mayor secreción total del cortisol. De manera similar, el AUCi del cortisol se relaciona positiva y significativamente con las estrategias de espíritu de lucha y evitación, lo cual indica que a mayor uso de estas estrategias, mayor reactividad del cortisol.

Tabla 2.

Análisis de correlación entre AUC de cortisol y variables psicológicas

\begin{tabular}{lll}
\hline Variable & AUCg & AUCi \\
& $\mathrm{rs}(\mathrm{p})$ & $\mathrm{rs}(\mathrm{p})$ \\
\hline Estrés & $-.593(.012)^{*}$ & $-.447(.072)$ \\
Optimismo & $.127(.628)$ & $-.022(.933)$ \\
Espíritu de lucha & $.642(.005)^{* *}$ & $.490(.046)^{*}$ \\
Desesperanza & $.133(.612)$ & $.172(.508)$ \\
Ansiedad & $-.183(.482)$ & $-.172(.508)$ \\
Fatalismo & $-.130(.620)$ & $-.002(.992)$ \\
Evitación & $.537(.026)^{*}$ & $.566(.018)^{*}$ \\
\hline
\end{tabular}

Nota: rs: correlación de Spearman, p: significancia estadística, ${ }^{* *} \mathrm{p}<.01,{ }^{*} \mathrm{p}<.05$ 
En cuanto a la relación entre las variables psicológicas se encontró relación negativa y significativa entre el estrés y el optimismo $\left(\mathrm{r}_{\mathrm{s}}=-.618, \mathrm{p}=.008\right)$, y entre el estrés y la estrategia de espíritu de lucha $\left(\mathrm{r}_{\mathrm{s}}=-.809, \mathrm{p}=.000\right)$. El optimismo se relacionó positiva y significativamente con espíritu de lucha $\left(\mathrm{r}_{\mathrm{s}}=.663 \mathrm{p}=.004\right)$.

Análisis de regresión

En el análisis de regresión múltiple con la AUCg de cortisol como variable dependiente, y el estrés y las estrategias de afrontamiento de espíritu de lucha y evitación cognitiva como variables independientes, se obtuvieron dos modelos (Tabla 3). En el primero, considerando la $\mathrm{R}$ ajustada, el espíritu de lucha explica un $40.5 \%$ de la varian- za del cortisol. En el segundo, la estrategia de espíritu de lucha junto con la evitación cognitiva explican un 57.8\% de la varianza de la concentración total del cortisol. Ambos modelos obtuvieron un valor de Durbin-Watson de 1.110, por los que se consideran adecuados.

De igual manera, en el análisis de regresión de la AUCi del cortisol, también se obtuvieron dos modelos: en el primero, considerando la $\mathrm{R}$ ajustada, el espíritu de lucha explica el $33.3 \%$ del incremento del cortisol, y en el segundo, el espíritu de lucha, junto con la evitación cognitiva, explican el 55.8\% de la varianza del incremento del cortisol. Ambos modelos obtuvieron un valor de Durbin-Watson de1.634, por los que se consideran adecuados.

Tabla 3.

Análisis de regresión de los niveles de cortisol

\begin{tabular}{|c|c|c|c|c|c|}
\hline Variable & R2aj & $\mathrm{F}$ & B & Beta & $\mathrm{T}$ \\
\hline \multicolumn{6}{|l|}{ AUCg } \\
\hline Modelo 1 & .405 & $11.89 * *$ & 6.403 & .665 & $3.449 * *$ \\
\hline \multicolumn{6}{|l|}{ Espíritu de lucha } \\
\hline \multicolumn{6}{|l|}{ Modelo 2} \\
\hline Espíritu de lucha & .578 & $7.13 *$ & ( & .000 & 3.001 \\
\hline \multicolumn{6}{|l|}{ Evitación } \\
\hline \multicolumn{6}{|l|}{ AUCi } \\
\hline Modelo 1 & .333 & $8.97^{* *}$ & 5.351 & .612 & $2.996^{* *}$ \\
\hline \multicolumn{6}{|l|}{ Espíritu de lucha } \\
\hline Modelo 2 & & & 4.588 & .525 & 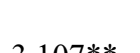 \\
\hline Espíritu de lucha & .558 & $8.66^{*}$ & 17.376 & .497 & $2.943^{*}$ \\
\hline Evitación & & & & & \\
\hline
\end{tabular}

Nota: AUCg: Área bajo la curva base, AUCi: Área bajo la curva incremento** $\mathrm{p}<.01,{ }^{*} \mathrm{p}<.05$

\section{DISCUSIÓN}

Los principales resultados de este estudio son un ritmo diurno de cortisol normal, con una mayor secreción de cortisol durante la mañana y una disminución a lo largo del día en las pacientes con cáncer de mama en los tres estadios. Estos resultados son diferentes a los publicados en otros estudios (Sephton et al., 2000; Touitou et al., 1996), en los que las pacientes presentan valores bajos, incrementos 0 decrementos irregulares de cortisol, evaluados en sangre y en saliva. Esta diferencia puede ser debida a que en estos estudios algunas pacientes presentaban metástasis o ya habían pasado por quimioterapia o tratamientos hormonales como el megestrol, que alteran el eje HPA reduciendo la secreción del cortisol (Morrow, Hickok, Andrews y Stern, 2002; Raeder et al., 2003). En este estudio, las pacientes fueron evaluadas previo al tratamiento de quimioterapia para control el efecto citotóxico sobre el funcionamiento del eje HPA.

Este punto es importante, ya que en pacientes con cáncer de ovario que solo tuvieron tratamiento de cirugía 
se encontró que entre seis y doce meses después, el ritmo diurno de cortisol se normaliza, lo cual se observa en una disminución en los niveles de cortisol de la mañana, tarde y noche (Schrepf et al., 2013). Esto sugiere que la alteración del ritmo diurno del cortisol en las pacientes con cáncer puede presentarse en fases específicas de la enfermedad, es decir, antes o después de cirugía o antes o después del tratamiento de quimioterapia.

A pesar de que las pacientes muestran un ritmo de cortisol normal, se observó que aquellas en el estadio III presentan mayor secreción en algunas muestras, al igual que las pacientes en estadio I, que presentan mayor secreción en el día 2. Esto es similar a lo reportado en otros estudios que muestran que pacientes con diferentes tipos de cáncer con altos niveles de cortisol por la mañana o por la noche presentan un ritmo diurno del cortisol normal (Lissoni et al., 2007; Mazzoccoli et al., 2010). Esto puede indicar que aunque los pacientes con cáncer tengan secreción de cortisol más alta, ello no necesariamente representa una alteración en el ritmo diurno del cortisol. Aunque no está claro si esta condición a largo plazo puede alterar el ritmo diurno del cortisol, sería importante realizar estudios de seguimiento con aquellas personas que presentaban valores superiores a lo normal.

Debido a la variabilidad en los enfoques de los análisis estadísticos utilizados en los diferentes estudios, hay dificultades para hacer comparaciones entre los resultados de los mismos (Powell, Liossi, Moss-Morris \& Schlotz, 2013). Sin embargo, a continuación se discuten los resultados obtenidos en cuanto a la relación entre variables psicológicas y la AUC de cortisol:

Al realizar el análisis de comparación del área bajo la curva del cortisol por estadios, no se observan diferencias significativas. Sin embargo, el estadio III tiene una media más alta que los estadios I y II. De manera similar, Weinrib et al., (2010) tampoco encontraron diferencias entre estadios tempranos y avanzados en la concentración del cortisol. Al comparar las variables psicológicas, solo se encontraron diferencias significativas en la variable de optimismo, siendo las pacientes en el estadio III las que presentan un optimismo más bajo. También presentaban mayor estrés, desesperanza y ansiedad, aunque estos no fueron significativos, lo cual puede reflejar la incertidumbre de no haber recibido ningún tipo de tratamiento a diferencia de los otros estadios.

En cuanto a la relación entre los niveles de cortisol y las variables psicológicas, se esperaba que la relación entre el estrés y los niveles de cortisol fuera positiva debido a que los estudios muestran que cuando el estrés percibido aumenta, también aumenta la secreción de cortisol (Fan et al., 2009; Schoofs, Hartmann \& Wolf, 2008); sin embargo, se obtuvo una dirección negativa. Otros estudios no encuentran relación entre el cortisol y variables como el distrés, estrés, depresión y ansiedad (Carlson et al., 2007; Porter et al., 2003, Septhon et al., 2000; Vedhara et al., 2003). Solo un estudio muestra una relación negativa entre el estrés y los niveles de cortisol, pero no fue significativa (Rosal, King, Yunsheng \& Reed, 2004).

Algunos estudios han sugerido la posibilidad de una relación negativa entre estrés y cortisol, explicando que debido a situaciones estresantes constantes, el organismo se habitúa, de manera que la secreción de cortisol disminuye (Fries, Hesse, Hellhammer \& Hellhammer 2005; Wüst, Federenko, VanRossum, Koper \& Hellhammer, 2005). Esto puede explicar la relación negativa entre el estrés y los niveles de cortisol, ya que aunque se evaluó el estrés percibido, las pacientes que participaron en este estudio ya habían experimentado varios estímulos estresantes como un diagnóstico de una enfermedad crónica y procedimientos invasivos como biopsia o mastectomía.

En cuanto a las estrategias de afrontamiento y los niveles de cortisol, el espíritu de lucha y la evitación cognitiva son las variables que más influyen en los niveles del cortisol. Hay pocos estudios que muestran la relación entre las estrategias de afrontamiento y los niveles de cortisol y los resultados son contradictorios (Sjögren, Leanderson \& Kristenson, 2006; O’Donnell, Badrick, Kumari \& Steptoe, 2008).

Desde un punto de vista psicológico, la dirección de la relación entre la estrategia de afrontamiento de espíritu de lucha y los niveles de cortisol es inesperada, ya que esta estrategia es considerada positiva, debido a que los pacientes que la utilizan tienen una visión optimista acerca del cáncer y lo toman como un reto y con la determinación de luchar contra él y no para permitir que se interrumpa su vida (Coyne \& Tennen, 2010).

Estos resultados se pueden explicar debido a que la evaluación de una condición como desafío conlleva una percepción de amenaza y una sensación de incertidumbre en cuanto a lo que pueda suceder en el futuro (Lazarus, 2000). Asimismo, las personas que tratan de manejar más efectivamente las situaciones estresantes son las que más se involucran, y esto genera un estrés potencial para sí mismos (Folkman \& Mozkowitz, 2004; Solberg, Segerstrom $\&$ Septhon, 2005).

Por su parte, la estrategia de evitación es más utilizada cuando se hace una valoración de amenaza, daño o pérdida (Bigatti, Steiner \& Miller, 2012) y, contrario a lo que se encontró en este estudio, se ha relacionado con mayor ansiedad y depresión (Costanzo, Lutgendorf, Rothrock y Anderson, 2006; Donovan- Kicken \& Caughlin, 2011), y no 
se encuentra relación con indicadores del cortisol (Dedert et al., 2012), por lo que es importante realizar más estudios para aclarar la relación obtenida.

Desde una perspectiva biológica, la relación entre los niveles de cortisol y las estrategias de afrontamiento de espíritu de lucha y evitación es consistente con la respuesta conductual clásica ante una condición amenazante, la cual consiste en luchar o huir (McEwen, 2007). Esto es importante ya que la activación del eje HPA produce la secreción del cortisol para movilizar y poner a disposición la energía del organismo y así pueda responder a las demandas del medio ambiente.

Las principales limitaciones de este estudio son la cantidad de participantes y la carencia de un grupo control sin cáncer. Otra limitación es la baja consistencia interna de la escala de optimismo y estrategias de afrontamiento. Para validar los datos en futuras investigaciones, se debe incluir un grupo control y otro grupo de pacientes en estadio IV de la enfermedad. Sin embargo, los hallazgos de este estudio son importantes debido a que se encontró relación entre variables psicológicas como el estrés y las estrategias de afrontamiento. Tal y como se señaló al principio, las relaciones encontradas en otros estudios son escasas o nulas.

Una implicación importante de los resultados de este estudio es la necesidad de revalorar la estrategia de espíritu de lucha y analizar su manejo en una intervención psicológica. Aunque se considera como una estrategia de afrontamiento positiva que genera un mejor ajuste ante la enfermedad de cáncer, entendiéndose este como menor ansiedad y depresión (Cordova et al., 2003), otros estudios sugieren que el fomentar el espíritu de lucha a veces es contraproducente en los pacientes, debido a que no saben cómo mantener esta actitud durante todo el proceso de la enfermedad, por lo que a veces puede ser desadaptativo (Spiegel, 2001; Watson, Haviland, Davidson y Bliss, 2000). También algunos estudios sugieren que la estrategia de evitación puede ser adaptativa y que permite la inhibición de la información que no es relevante para la meta (Master et al., 2009; Putman y Roelofs, 2011). Esto indica que se deben profundizar los estudios sobre la interacción entre estas variables.

\section{REFERENCIAS}

Abercrombie, H., Giese-Davis, J., Sephton, S., Epel, E., Turner-Cobb, J. \& Spiegel, D. (2003). Flattened cortisol rhythms in metastatic breast cancer patients. Psychoneuroendocrinology, 29, 1082-1092.

Barroilhet, S., Forjaz, M. \& Garrido, E. (2005). Conceptos, teorías y factores psicosociales en la adaptación al cáncer. Actas Españolas de Psiquiatría, 33(6), 390-397.
Bigatti, S., Steiner, J. \& Miller, K. (2012). Cognitive Appraisals, Coping and Depressive Symptoms in Breast Cancer Patients. Stress and Health, 28, 355-361.

Carlson, L., Campbell, T., Garland, S. \& Grossman, P. (2007). Associations among salivary cortisol, melatonin, catecholamines, sleep quality and stress in women with breast cancer and healthy controls. Journal of Behavioral Medicine, 30(1), 45-58.

Cohen, S., Kamarak, T. \& Mermelstein, R. (1983). A global measure of perceived stress. Journal of Health and Social Behavior, 24, 385-396.

Cordova, M., Giese-Davis, J., Golant, M., Kronnenwetter, C., Chang, V., McFarlin, S. \& Spiegel, D. (2003). Mood disturbance in community cancer support groups: The role of emotional suppression and fighting spirit. Journal of Psychosomatic Research, 55, 5, 461-467.

Costanzo, E., Lutgendorf, S., Rothrock, N. \& Anderson, B. (2006). Coping and quality of life among women extensively treated for gynecologic cancer. Psycho-Oncology, 15, 132-142.

Coyne, J. \& Tennen, H. (2010). Positive Psychology in Cancer Care: Bad Science, Exaggerated Claims and Unproven Medicine. Annals of Behavioral Medicine, 39, 16-26.

Dedert, E., Lush, E., Chagpar, A., Dhabhar, F., Segestrom, S., Spiegel, D., Dayyat, E., Daup, M., McMasters, K. \& Sephton, S. (2012). Stress, Coping, and Circadian Disruption Among Women Awaiting Breast Cancer Surgery. Annals of Behavioral Medicine, 44(1), 10-20.

Dettenborn, L., James, G., Valdimarsdottir, H., Montgomery, G. \& Bovbjerg, D. (2006). Breast cancer-specific intrusions are associated with increased cortisol responses to daily life stressors in healthy women without personal or family histories of breast cancer. Journal of Behavioral Medicine, 29(5), 477-485.

Donovan-Kicken, E. \& Caughlin, J. (2011). Breast cancer patients' topic avoidance and psychological distress: The mediating role of coping. Journal of Health Psychology, 16(4): 596-606.

Fan, Y., Tang, Y., Lu, Q., Feng, S., Yu, Q., Sui, D., Zhao, Q., Ma, Y. \& Li, S. (2009). Dynamic changes in salivary cortisol and secretory immunoglobulin A response to acute stress. Stress and Health, 25, 189-194.

Fekedulegn, D. Andrew, M., Burchfiel, C., Violanti, J., Hartley, T., Charles, L.E. \& Miller D.B. (2007). Area under the curve and other summary indicators of repeated waking cortisol measurements. Psychosomatic Medicine, 69, 651-665.

Fischbach, F. \& Dunning, M. (2009). A manual of laboratory and diagnostic test. Ed 8. China: Wolters Kluwer Health/ Lippincott Williams and Wilkins.

Fries, E., Hesse, J., Hellhammer, J. \& Hellhammer, D. (2005). A new view on hypocortisolisms. Psychoneuroendocrinology, 30(10), 1010-1016. 
Folkman, S. \& Moskowitz, J. (2004). COPING: Pitfalls and Promise. Annual Review of Psychology, 55(1), 745-774. doi:10.1146/annurev.psych.55.090902.141456

Garland, M., Lavelle, E., Doherty, D., Golden-Mason, L., Fitzpatrick, P., Hill, A., Walsh, N. \& O' Farrely, C. (2004). Cortisol does not mediate the suppressive effects of psychiatric morbidity on natural killer cell activity: A cross-sectional study of patients with early breast cancer. Psychological Medicine, 34, 481- 490.

González, M. \& Landero, R. (2007). Factor structure of the perceived stress scale (PSS) in a sample from México. The Spanish Journal of Psychology, 10(1): 199-206.

Haus, E., Dumitriu, L., Nicolau, G., Bogola, S. \& SackaettLunden, L. (2001). Circadian Rhythms of basic fibroblast growth factor (bFGF), epidermal growth factor (EGF), insulin-like growth factor-1 (IGG-1), insulin-like growth factor binding protein-3 (IGFBP-3) cortisol and melatonin in women with breast cancer. Chronobiology International, 18(4), 709-727.

Kronfol, Z., Nair, M., Zhang, Q., Hill, E. \& Brown, M. (1997). Circadian immune measures in healthy volunteers: Relationship to hypothalamic-pituitary-adrenal axis hormones and sympathetic neurotransmitters. Psychosomatic Medicine, 59, 42-50.

Lang, E., Berbaum, K. \& Lutgendorf, S. (2009). Large-Core Breast Biopsy: Abnormal Salivary Cortisol Profiles Associated with Uncertainty of Diagnosis. Radiology, 250(3), 631-637.

Lazarus, R. (2000). Estrés y Emoción: Manejo e implicaciones en nuestra salud. Bilbao: Descleé de Brower.

Lissoni, P., Brivio, F., Fumagalli, L., Messina, G., Secreto, G., Romelli, B., \& Brera, G. (2007). Immune and endocrine mechanisms of advanced cancer-related hypercortisolemia. In vivo, 21(4), 647-650.

Master, S., Amodio, D., Stanton, A., Yee, C., Hilmert, C. \& Taylor, S. (2009). Neurobiological Correlates of Coping through Emotional Approach. Brain, Behavior and Immunity, 23(1), 27-35.

McEwen, B. (2007). Physiology and Neurobiology of Stress and Adaptation: Central Role of the Brain. Physiological Review, 87, 873-904, doi:10.1152/physrev.00041.2006.

Mazzoccoli, G., Giuliani, F. \& Sothern, R. (2012). Determination of whole body circadian phase in lung cancer patients: melatonin vs. cortisol. The International Journal of Cancer Epidemiology, Detection, and Prevention, 36, 46-53.

Mazzoccoli, G., Tarquini, R., Durfot, T. \& Francois, J. (2011). Chronodisruption in lung cancer and possible therapeutic approaches. Biomedicine and Pharmacotherapy, 65, 500508.

Mazzoccoli, G., Vendemiale, G., De Cata, A., Carughi, S. \& Tarquini, R. (2010). Altered time structure of neuro-endocrine-immune system function in lung cancer patients. BMC Cancer, 10, 314. Doi:10.1186/1471-2407-10-314
Morrow, G., Hickok, J., Andrews, P. \& Stern, R. (2002). Reduction in serum cortisol after platinum based chemotherapy for cancer: A role for the HPA axis in treatment-related nausea. Psychophysiology, 39(4), 491-495.

Moscoso, M. (2009). De la mente a la célula: Impacto del estrés en psiconeuroinmunoendocrinología. LIBERABIT, 15(2), 143-152.

Nakaya, N., Fukudo, S., Akizuki, N., Yoshikawa, E., Kobayakawa, M., Fujimori, M., ... \& Uchitomi, Y. (2005). Twenty-four-hour urinary cortisol levels before complete resection of non-small cell lung cancer and survival. Acta Oncologica, 44(4), 399-405.

Otero, J., Luengo, A., Romero, E., Gómez, J. \& Castro, C. (1998). Psicología de personalidad. Manual de prácticas. Barcelona: Ariel Practicum.

O’Donnell, K., Badrick, E., Kumari, M. \& Steptoe, A. (2008). Psychological coping styles and cortisol over the day in healthy older adults. Psychoneuroendocrinology 33(5), 601-611.

Porter, L., Mishel, M., Neelon, V., Belyea, M., Pisano, E. \& Scott, M. (2003). Cortisol levels and responses to mammography screening in breast cancer survivors: A pilot study. Psychosomatic Medicine, 65, 842-848.

Powell, D., Liossi, C., Moss-Morris, R. \& Schlotz, W. (2013). Unstimulated cortisol secretory activity in everyday life and its relationship with fatigue and chronic fatigue syndrome: A systematic review and subset meta-analysis. Psychoneuroendocrinology, 38, 2405-2422.

Pruessner, J., Kirshbaum, C., Meinlschmid, P. \& Hellhammer, D. (2003). Two formulas for computation of the area under the curve represent measures of total hormone concentration versus time-dependent change. Psychoneuroendrocrinology, 28, 916-931.

Putman, P. \& Roelofs, K. (2011). Effects of single cortisol administrations on human affect reviewed: Coping with stress through adaptive regulation of automatic cognitive processing. Psychoneuroendocrinology, 36, 439-448.

Raedler, T., Jahn, H., Goedeken, B., Gescher, D., Kellner, M. \& Wiedemann, K. (2003). Acute effects of megestrol on the hypothalamic-pituitary-adrenal axis. Cancer Chemotherapy and Pharmacology, 52, 482-486. doi 10.1007/s00280003-0697-6.

Rosal, M., King, J., Yunsheng, M. \& Reed, G. (2004). Stress, Social Support, and Cortisol: Inverse Associations. Behavioral Medicine, 30, 11-21.

Salimetrics, LLC. (2012). Collecting Saliva. Recuperado el 30 de agosto de 2012, desde http://www.salimetrics.com/literature

Salimetrics, LLC. (2013). Salivary Cortisol. Enzime Immunoassay Kit. Recuperado el 7 de enero de 2014 desde http:// www.salimetrics.com/documents/1-3002.pdf

Scheier, M., Carver, C. \& Bridges, M. (1994). Distinguishing optimism from neuroticism (and trait anxiety, self-mastery 
and self-esteem): A reevaluation of the Life Orientation Test. Journal of Personality and Social Psychology, 67(6), 1063-1078.

Schrepf, A., Clevenger, L., Christensen, D., DeGeest, K., Bender, D., Ahmed, A., ... \& Lutgendorf, S. K. (2013). Cortisol and inflammatory processes in ovarian cancer patients following primary treatment: Relationships with depression, fatigue, and disability. Brain, behavior, and immunity, 30, S126-S134.

Schoofs, D., Hartmann, R. \& Wolf, O. (2008). Neuroendocrine stress response to an oral academic examination: No strong influence of sex, repeated participation and personality traits. Stress, 11(1), 52-61.

Spiegel, D. (2001). Mind matters: Coping and cancer progression. Journal of Psychosomatic Research, 50, (5), 287-290.

Septhon, S., Lush, E., Dedert, E., Floyd, A., Rebholz, W., Dhabhar, F., Spiegel, D. \& Salmon, P. (2012). Diurnal cortisol rhythm as a predictor of lung cancer survival. Brain, Behavior and Immunity, 30, 163-170. DOI:10.1016/j. bbi.2012.07.019

Sephton, S., Sapolsky, R., Kraemer, H. \& Spiegel, D. (2000). Diurnal cortisol rhythm as a predictor of breast cancer survival. Journal of the National Cancer Institute, 92(12), 9941000.

Sephton, S. \& Spiegel, D. (2003). Circadian disruption in cancer: A neuroendocrine-immune pathway from stress to disease? Brain, Behavior and Immunity, 17, 321-328.

Solberg, L., Segerstrom, S. \& Septhon, S. (2005). Engagement and Arousal: Optimism's Effects During a Brief Stressor. Personality and Social Psychology Bulletin, 31(1), 111-120.

Sjögren, E., Leanderson, P. \& Kristenson, M. (2006). Diurnal cortisol levels and relations to psychosocial factors in a population sample of middle-age Swedish men and women. International Journal of Behavioral Medicine, 13(3), 193-200.

Taylor, S., Burklund, L., Eisenberger, N., Lehman, B., Hilmert, C. \& Lieberman, M. (2008). Neural bases of moderation of cortisol stress responses by psychosocial resources. Journal of Personality and Social Psychology, 95(1), 197-211.

Touitou, Y., Bogdan, A., Lévi, F., Benavides, M. \& Auzéby, A. (1996). Disruption of the circadian patterns of serum cortisol in breast and ovarian cancer patients: Relationships with tumor marker antigens. British Journal of Cancer, 74, 1248-1252.

Touitou, Y., Lévi, F., Bogdan, A., Benavides, M., Bailleul, F. \& Misset, J. (1995). Rhythm alteration in patients with metastatic breast cancer and poor prognostic factors. Journal Cancer Research Clinic Oncology, 121, 181-188.

Vedhara, K., Miles, J., Bennett, P., Plummer, S., Tallon, D., Brooks, E., ... \& Farndon, J. (2003). An investigation into the relationship between salivary cortisol, stress, anxiety and depression. Biological psychology, 62(2), 89-96.

Vedhara, K., Tuin, J., Miles, J., Sanderman, R. \& Ranchor, A. (2006). Psychosocial factors associated with indices of cortisol production in women with breast cancer and controls. Psychoneuroendocrinology, 31, 299-311.

Watson, M., Greer, S., Young, J., Inayat, Q., Burgesss, G. \& Robertson, B. (1988). Development of questionnaire measure of adjustment to cancer: the MAC scale. Psychological Medicine, 18, 203-206.

Watson, M., Haviland, J., Davidson, J. \& Bliss, J. (2000). Fighting spirit in patients with cancer. Lancet, 355, 4, 848,

Weinrib, A. Z., Sephton, S. E., DeGeest, K., Penedo, F., Bender, D., Zimmerman, B., ... \& Lutgendorf, S. K. (2010). Diurnal cortisol dysregulation, functional disability, and depression in women with ovarian cancer. Cancer, 116(18), 4410-4419.

Wüst, S., Federenko, I., Van Rossum, E., Koper, J. \& Hellhammer, D. (2005). Habituation of cortisol responses to repeated psychosocial stress-further characterization and impact of genetic factors. Psychoneuroendocrinology, 30(2), 199211.

Young, C. \& Welsh, J. (2005). Stress, Health and Disease: A Review. Cell Science, 2(2), 132-158. 